2. M. Gerstenhaber, The cohomology structure of an associative ring, Ann. of Math. (2) 78 (1963), 267-288.

3. - On the deformation of rings and algebras, Ann. of Math. (2) 79 (1964), 59-103.

4. A. Nijenhuis, Jacobi-type identities for bilinear differential concomitants of certain tensor fields, Kon. Nederl. Akad. Wetensch. Proc. A58-Indag. Math. 17 (1955), 390403.

5. A. Nijenhuis and R. W. Richardson, Cohomology and deformations in graded Lie algebras, Bull. Amer. Math. Soc. 72 1(1966), 1-29.

6. - Deformations of homorphisms of Lie groups and Lie algebras, Bull. Amer. Math. Soc. 73 (1967), 175-179. 106.

7. - Deformations of Lie algebra structures, J. Math. Mech. 17 (1967), 89-

8. R. W. Richardson, A rigidity theorem for subalgebras of Lie and associative algebras, Illinois J. Math. 11 (1967), 92-110.

9. A. Nijenhuis, The graded Lie algebras of an algebra, Kon. Nederl. Akad. Wetensch. Proc.-Indag. Math. (to appear).

University of Pennsylvania

\title{
BOUNDED APPROXIMATION BY POLYNOMIALS WITH RESTRICTED ZEROS ${ }^{1}$
}

\author{
BY CHARLES KAM-TAI CHUI
}

Communicated by R. C. Buck, July 19, 1967

1. Introduction. Let $C$ be a rectifiable Jordan curve, $D$ its interior. A sequence of polynomials $P_{n}(z)$ is said to converge boundedly to a function $f(z)$ in $D$, or equivalently, $f(z)$ is said to be boundedly approximated by the polynomials $P_{n}(z)$ in $D$, if $\sup \left\{\left|P_{n}(z)\right|: z \in D\right\}$ is bounded as a function of $n$, and $\left\{P_{n}(z)\right\}$ converges to $f(z)$ throughout $D$. It is known [1], [6] that $f(z)$ can be boundedly approximated by polynomials in $D$ if and only if $f(z)$ is a bounded holomorphic function in $D$. In this paper we consider the more delicate bounded approximation problem in which the zeros of the polynomials are required to lie on the boundary $C$. Polynomials whose zeros lie on $C$ are called $C$-polynomials.

A different kind of appproximation by $C$-polynomials was studied by G. R. MacLane [5]. He proved that if $f(z)$ is holomorphic and zero free in $D$, then there exists a sequence of $C$-polynomials which converges to $f(z)$ uniformly on every compact subset of $D$. This result was later extended by J. Korevaar [3] and his students [4] to more general sets $D$.

1 This is an announcement of the author's doctoral thesis at the University of Wisconsin written under the direction of Professor Jacob Korevaar. The author wishes to acknowledge partial support from National Science Foundation grant GP-4106. 
It is obvious that the limit function of a boundedly convergent sequence of $C$-polynomials is bounded, holomorphic and zero free in $D$, unless it is the zero function. When $C$ is the unit circle $\{z:|z|=1\}$, it turns out (somewhat unexpectedly) that the converse is true.

MAIN THEOREM. Every zero free, bounded holomorphic function in the open unit disc can be boundedly approximated by polynomials whose zeros lie on the unit circumference.

2. Outline of proof. Throughout this section, $D$ will denote the open unit disc $\{z:|z|<1\}$ and $C$ the unit circle. The proof can be divided into two main steps.

Step I. Preliminary approximation. The following representation is an easy consequence of Herglotz's theorem for nonnegative harmonic functions [2].

Lemma 1. Let $f(z)$ be holomorphic and zero free in $D$, and such that $f(0)=1$ and $\sup |f(z)|=M<+\infty$. Then there exists a nondecreasing real-valued function $\nu(t)$ in $[0,2 \pi]$, with $\nu(0)=0$ and $\nu(2 \pi)=2 \log M$, such that

$$
f(z)=\exp \left\{\int_{0}^{2 \pi} \frac{-z e^{-i t}}{1-z e^{-i t}} d \nu(t)\right\} \quad \text { for } z \in D .
$$

Formula (1) indicates the importance of the following zero free, bounded holomorphic function in $D$ :

$$
f(z)=\exp \{-z /(1-z)\} \text {. }
$$

Our proof of the main theorem depends on bounded approximation of this special function. We try to approximate $f(z)$ by expressions of the form

$$
S_{n}(z)=\prod_{k=1}^{n}(1-z \exp (-i 2 \pi k / n))^{\alpha_{n, k},}
$$

where $\alpha_{n, k} \geqq 0$ for $k=1, \cdots, n$, and

$$
\max _{1 \leqq t \leqq n} \alpha_{n, k} \rightarrow 0 \text {. }
$$

Suppose that as $n \rightarrow \infty, S_{n}(z) \rightarrow \exp \{-z /(1-z)\}$ uniformly on the closed subsets of $D$. Taking the power series of the principal values of the logarithms, we find that, in the same sense,

$$
-\sum_{\nu=1}^{\infty} \frac{1}{\nu}\left\{\sum_{k=1}^{n} \alpha_{n k} \exp (-i 2 \pi k \nu / n)\right\} z^{\nu} \rightarrow-\sum_{\nu=1}^{\infty} z^{\nu}
$$


Hence, we must have coefficientwise convergence:

$$
\sum_{k=1}^{n} \alpha_{n, k} \exp (-i 2 \pi k \nu / n) \rightarrow \nu \text { for } \nu=1,2, \cdots
$$

We begin by considering the first $p$ of these relations,

$$
\sum_{k=1}^{n} \alpha_{n, k}^{(p)} \exp (-i 2 \pi k \nu / n) \rightarrow \nu \text { for } \nu=1, \cdots, p .
$$

Let $n>2 p$. A convenient (but not obvious) solution of (4) is given by

$$
\alpha_{n, k}^{(p)}=\sum_{j=1}^{p}(2 j / n)(1-j / p)(1+\cos (2 \pi j k / n)) ;
$$

it is clear that for each $k=1, \cdots, n$,

$$
0 \leqq \alpha_{n, k}^{(p)}<p^{2} / n
$$

It can be shown that when we let $n$ tend to $\infty$ through the positive integers, and let $p$ tend to $\infty$ in such a way that $p^{2} / n$ tends to 0 , then

$$
\sum_{k=1}^{n} \alpha_{n, k}^{(p)} \log (1-z \exp (-i 2 \pi k / n)) \rightarrow \frac{-z}{1-z}
$$

for each $z$ in $D$.

It can also be shown that for $z=r e^{i \theta}$,

$$
\begin{aligned}
\max _{|z| \leqq 1} & \sum_{k=1}^{n} \alpha_{n, k}^{(p)} \log |1-z \exp (-i 2 \pi k / n)| \\
& \leqq \max _{0 \leqq \theta \leqq 2 \pi}\left\{1 / 2-F_{p}(\theta)+p^{2} / n+p^{3} / n^{2}\right\}<1 / 2+2 p^{2} / n,
\end{aligned}
$$

where $F_{p}(\theta)$ denotes the Fejér kernel of order $p$. Thus as $n \rightarrow \infty$ and $p^{2} / n \rightarrow 0$,

$$
\max _{|z| \leqq 1}\left|\prod_{k=1}^{n}(1-z \exp (-i 2 \pi k / n)) \alpha_{n, k}^{(p)}\right| \leqq \exp (1 / 2+\epsilon) .
$$

Using the above result and Lemma 1, we obtain

LEMMA 2. Let $f(z)$ be any zero free holomorphic function in $D$ such that $f(0)=1$ and sup $|f(z)|=M<+\infty$. Then for any given $\epsilon>0$, there exists a sequence of functions $S_{n}(z)$ of the form indicated in (2) and (3) such that $\left\{S_{n}(z)\right\}$ converges to $f(z)$ in $D$, and

$$
\max _{|z| \leqq 1}\left|S_{n}(z)\right| \leqq M^{1+\bullet} \text {. }
$$


Step II. Bounded approximation by C-polynomials. Let $S_{n}(z)$ be functions of the form indicated in (2) and (3). For each $n$, we will construct a sequence of $C$-polynomials

$$
P_{m+n}(z, n)=\prod_{j=1}^{m}\left(1-z \exp \left(-i t_{j}\right)\right) \prod_{k=1}^{n}\left(1-z \exp \left(-i \theta_{k}\right)\right)
$$

which converges to $S_{n}(z)$ in $D$, and is such that

$$
\max _{|z| \leqq 1}\left|P_{m+n}(z, n)\right| \leqq e^{20} \max _{|z| \leqq 1}\left|S_{n}(z)\right|
$$

for all large $m$.

Set $\sum_{k=1}^{n} \alpha_{n, k}=\alpha$ where we assume that $0 \leqq \alpha_{n, k}<1$. The numbers $t_{j}=t_{j}(m), j=1, \cdots, m$, and $\theta_{k}=\theta_{k}(m), k=1, \cdots, n$, are defined by the following procedure

$$
\begin{gathered}
t_{1}=\frac{\left(2-\alpha_{n, n}\right) \pi}{m+n-\alpha}, \\
t_{2}=t_{1}+\frac{2 \pi}{m+n-\alpha}, \cdots, t_{j_{1}}=t_{j_{1}-1}+\frac{2 \pi}{m+n-\alpha},
\end{gathered}
$$

where $j_{1}$ is determined by the inequality $t_{j_{1}}<2 \pi / n \leqq t_{j_{1}}+2 \pi /(m+n-\alpha)$;

$$
\begin{gathered}
t_{j_{1}+1}=t_{j_{1}}+\frac{2\left(2-\alpha_{n, 1}\right) \pi}{m+n-\alpha}, \\
t_{j_{1}+2}=t_{j_{1}+1}+\frac{2 \pi}{m+n-\alpha}, \cdots, t_{j_{2}}=t_{j_{2}-1}+\frac{2 \pi}{m+n-\alpha},
\end{gathered}
$$

where $j_{2}$ is determined by the inequality $t_{j_{2}}<4 \pi / n \leqq t_{j_{2}}+2 \pi /(m+n-\alpha)$;

$$
\begin{gathered}
t_{j_{n-1}+1}=t_{j_{n-1}}+\frac{2\left(2-\alpha_{n, n-1}\right) \pi}{m+n-\alpha}, \\
t_{j_{n-1}+2}=t_{j_{n-1}+1}+\frac{2 \pi}{m+n-\alpha}, \cdots, t_{j_{n}}=t_{j_{n-1}}+\frac{2 \pi}{m+n-\alpha} ;
\end{gathered}
$$

here $j_{n}$ is determined by the inequality $t_{j_{n}}<2 \pi \leqq t_{j_{n}}+2 \pi /(m+n-\alpha)$; it turns out that $j_{n}=m$ and that

$$
t_{j_{n}}=t_{m}=2 \pi-\left(2-\alpha_{n, n}\right) \pi /(m+n-\alpha) ;
$$

finally,

$$
\theta_{1}=0 \text { and } \theta_{k}=\frac{1}{2}\left(t_{j_{k-1}}+t_{j_{k-1}+1}\right), \quad k=2, \cdots, n \text {. }
$$


It can be shown that for each $n$, the sequence $\left\{P_{m+n}(z, n)\right\}$ converges to $S_{n}(z)$ in $D$. That $P_{m+n}(z, n)$ satisfies (7) is a consequence of the following lemma.

LEMMA 3. For each $m=2,3, \cdots$, let $\tau_{k}=\tau_{k}(m), k=1, \cdots, m$, be points on $[0,2 \pi]$ such that $0<\tau_{1}<\cdots<\tau_{m} \leqq 2 \pi$, and, setting $\tau_{m+1}=\tau_{1}+2 \pi$,

$$
\frac{\max _{1 \leqq k \leqq m}\left(\tau_{k+1}-\tau_{k}\right)}{\min _{1 \leqq k \leqq m}\left(\tau_{k+1}-\tau_{k}\right)} \leqq A .
$$

Also, let $\omega_{k}=\omega_{k}(m)$ and $\alpha_{k}=\alpha_{k}(m)$ be defined as

$$
\omega_{k}=(1 / 2)\left(\tau_{k+1}+\tau_{k}\right), \quad \alpha_{k}=(m / 2 \pi)\left(\tau_{k+1}-\tau_{k}\right) .
$$

Finally, let

$$
R_{m}(z)=\prod_{k=1}^{m}\left(1-z \exp \left(-i \omega_{k}\right)\right)^{\alpha_{h}} .
$$

Then for all large $m$,

$$
\max _{|z| \leqq 1}\left|R_{m}(z)\right| \leqq \exp \left(19 A^{3}\right) .
$$

Now, suppose that $f(z)$ is as given in Lemma 1 . We can find functions $S_{n}(z)$ of the form indicated in (2) and (3) which approximate $f(z)$ and satisfy (5). For each $n$, a sequence of $C$-polynomials $P_{m+n}(z, n)$ can be constructed to approximate $S_{n}(z)$, while $P_{m+n}(z, n)$ satisfies (7). Hence, for any given $\epsilon>0$, any $n$, and sufficiently large $m$,

$$
\max _{|z| \leqq 1}\left|P_{m+n}(z, n)\right| \leqq e^{20} M^{1+e} \text {. }
$$

It is not difficult to see that we can select a sequence of $C$-polynomials $P_{n}(z)$ from the family $\left\{P_{m+n}(z, n)\right\}$ in (8) which converges to $f(z)$ throughout $D$. This will complete the proof of the main theorem.

3. Extension to more general regions. Let $D$ be a bounded, simply connected domain in the z-plane whose boundary is a rectifiable Jordan curve $C$. Let $D^{\infty}$ denote the complement of the closure of $D$ with respect to the extended $z$-plane, and let $z=\Phi(w), \Phi(1)=\zeta_{0} \in C$, $\Phi(0)=\infty$, map the open unit disc $\{w:|w|<1\}$ conformally and homeomorphically onto $D^{\infty}$. It is clear that $\Phi(w)$ can be extended to a topological map (also denoted by $\Phi(w)$ ) on the closed disc $\{w:|w| \leqq 1\}$. Set $\phi(t)=\Phi\left(e^{i t}\right), 0 \leqq t \leqq 2 \pi$, and assume, without loss of generality, that $0 \in D$. We have the following representation formula [5]. 
LEMMA 4. Let $f(z)$ be holomorphic and zero free on clos $D$, and such that $f(0)=1$. Then there exists a real-valued function $\mu(t)$ which is analytic for real $t$ and of period $2 \pi$, with $\mu(0)=0$, such that

$$
\log f(z)=\int_{0}^{2 \pi} \log \left(1-\frac{z}{\phi(t)}\right) d \mu(t) \quad \text { for } z \in D .
$$

Here the logarithms denote the branches which vanish at $z=0$.

Using this lemma and methods similar to those indicated in Step II of $\$ 2$, we obtain the following result.

THEOREM 2. Let $C$ be so smooth that its parametric representation $\zeta=h(s)$, where $s$ denotes arc length, has a Hölder continuous derivative. Let $f(z)$ be holomorphic and zero free in the closure of $D$. Then $f(z)$ can be boundedly approximated by $C$-polynomials in $D$.

\section{REFERENCES}

1. O. J. Farrell, On approximation by polynomials to a function analytic in a simply connected region, Bull. Amer. Math. Soc. 41 (1934), 707-711.

2. K. Hoffman, Banach spaces of analytic functions, Prentice-Hall, Englewood Cliffs, N. J., 1962, p. 67.

3. J. Korevaar, Asymptotically neutral distributions of electrons and polynomial approximation, Ann. of Math. (2) 80 (1964), 403-410.

4. - Limits of polynomials whose zeros lie in a given set, Proc. Amer. Math. Soc. Summer Institute on Entire Functions and Related Parts of Analysis, 1966.

5. G. R. MacLane, Polynomials with zeros on a rectifiable Jordan curve, Duke Math. J. 16 (1949), 461-477.

6. L. A. Rubel and A. L. Shields, Bounded approximation by polynomials, Acta Math. 112 (1964), 145-162.

UNIVERSITY OF WISCONSIN AND

University of California at San Diego 